\title{
EXTENSION OF SOME CLASSICAL SUMMATION THEOREMS FOR THE GENERALIZED HYPERGEOMETRIC SERIES WITH INTEGRAL PARAMETER DIFFERENCES
}

\author{
ARJUn K. RATHIE AND R. B. PARIS
}

\begin{abstract}
We derive extensions of the classical summation theorems of Kummer and Watson for the generalized hypergeometric series where $r$ pairs of numeratorial and denominatorial parameters differ by positive integers. The results are obtained with the help of a generalization of Kummer's second summation theorem for the ${ }_{2} F_{1}$ series given recently by Rakha and Rathie [Integral Transforms and Special Functions, 22, 823-840 (2011)] together with generalizations of the Euler transformations for the ${ }_{r+2} F_{r+1}(z)$ function. A few interesting special cases are also presented.
\end{abstract}

Mathematics subject classification (2010): Primary 33C20, Secondary 33C15.

Keywords and phrases: Generalized hypergeometric series, generalized Gauss and Kummer summation theorems, generalized Watson summation theorem.

\section{REFERENCES}

[1] J. Choi, A. K. Rathie And H. M. SRivastava, A generalization of a formula due to Kummer, Integral Transforms and Special Functions 22, 851-859, 2011.

[2] C. Fox, The expression of hypergeometric series in terms of similar series, Proc. London Math. Soc. 26, 201-210, 1927.

[3] P. W. KARLSSON, Hypergeometric functions with integral parameter differences, J. Math. Phys. 12, 270-271, 1971.

[4] Y. S. Kim, M. A. RAKHA AND A. K. RATHIE, Extensions of certain classical summation theorems for the series ${ }_{2} F_{1},{ }_{3} F_{2}$ and ${ }_{4} F_{3}$ with applications in Ramanujan's summations, Int. J. Math. \& Math. Sci., Article ID 309503, 26 pages, 2010.

[5] Y. S. Kim, A. K. RATHie, U. PANDEY AND R. B. PARIS, Some summation formulas for the hypergeometric series ${ }_{r+2} F_{r+1}\left(\frac{1}{2}\right)$, Hacettepe J. Math. Stat. 42, 281-287, 2013.

[6] Y. S. KIM, A. K. RATHIE AND R. B. PARIS, An extension of Saalschütz's summation theorem for the series ${ }_{r+3} F_{r+2}$, Integral Transforms and Special Functions 24 (11), 916-921, 2013.

[7] E. E. KuMmER, Über die hypergeometrische Reihe

$$
1+\frac{\alpha \cdot \beta}{1 \cdot \gamma} x+\frac{\alpha(\alpha+1) \cdot \beta(\beta+1)}{1 \cdot 2 \cdot \gamma(\gamma+1)} x^{2}+\frac{\alpha(\alpha+1)(\alpha+2) \cdot \beta(\beta+1)(\beta+2)}{1 \cdot 2 \cdot 3 \cdot \gamma(\gamma+1)(\gamma+2)} x^{3}+\cdots,
$$

J. Reine Angew. Math. 15, 39-93 and 127-172, 1836.

[8] J. L. Lavoie, F. Grondin AND A. K. RathiE, Generalizations of Watson's theorem on the sum of $a_{3} F_{2}$, Indian J. Math. 34, 23-32, 1992.

[9] J. L. Lavoie, F. Grondin And A. K. Rathie, Generalizations of Dixon's theorem on the sum of a ${ }_{3} F_{2}$, Math. Comp. 62, 267-276, 1994.

[10] J. L. Lavoie, F. GRondin AND A. K. RATHIE, Generalizations of Whipple's theorem on the sum of $a_{3} F_{2}$, J. Comput. Appl. Math. 72, 293-300, 1996.

[11] Y. L. LuKe, Mathematical Functions and Their Approximations, Academic Press, New York, 1975.

[12] R. S. MaIER, $P$-symbols, Heun identities, and ${ }_{3} F_{2}$ identities, Contemp. Math. 471, 139-159, 2008. 
[13] A. R. MILlER, Certain summation and transformation formulas for generalized hypergeometric series, J. Comp. Appl. Math. 231, 964-972, 2009.

[14] A. R. Miller AND R. B. PARIS, Certain transformations and summations for the generalized hypergeometric series with integral parameter differences, Integral Transforms and Special Functions, 22, 67-77, 2011.

[15] A. R. Miller AND R. B. PARIS, Euler-type transformations for the generalized hypergeometric function ${ }_{r+2} F_{r+1}(x)$, Zeitschrift Angew. Math. Phys. 62, 31-45, 2011.

[16] A. R. Miller AND R. B. PARIS, On a result related to transformations and summations of generalized hypergeometric series, Math. Communications 17, 205-210, 2012.

[17] A. R. MILleR AND R. B. PARIS, Transformation formulas for the generalized hypergeometric function with integral parameter differences, Rocky Mountain J. Math. 43, 291-327, 2013.

[18] A. R. Miller AND H. M. SRIVASTAVA, Karlsson-Minton summation theorems for the generalized hypergeometric series of unit argument, Integral Transforms and Special Functions 21, 603-612, 2010.

[19] M. A. RAKHA AND A. K. RATHIE, Generalizations of classical summation theorems for the series ${ }_{2} F_{1}$ and ${ }_{3} F_{2}$ with applications, Integral Transforms and Special Functions 22, 823-840, 2011.

[20] A. K. RATHIE AND R. B. PARIS, An extension of the Euler-type transformation for the ${ }_{3} F_{2}$ series, Far East. J. Math. Sci. 27, 43-48, 2007.

[21] L. J. Slater, Generalized Hypergeometric Functions, Cambridge University Press, Cambridge, 1966.

[22] H. M. SRIVASTAVA, Generalized hypergeometric functions with integral parameter differences, Nederl. Akad. Wetensch. Indag. Math. 35, 38-40, 1973. 